\title{
Various Shapes of Soft Palate: A Lateral Cephalometric Study
}

\author{
${ }^{1}$ B N Praveen, ${ }^{2}$ Sunita Amrutesh, ${ }^{3}$ Sumona Pal, ${ }^{4}$ Shubhasini AR, ${ }^{4}$ Syed Vaseemuddin \\ ${ }^{1}$ P rofessor, Department of Oral Medicine and Radiology, KLE Society's Institute of Dental Sciences, Bengaluru, Karnataka, India \\ ${ }^{2}$ P rofessor and Head, Department of Oral Medicine and Radiology, KLE Society's Institute of Dental Sciences, Bengaluru, Karnataka, India \\ ${ }^{3}$ P ostgraduate Student, Department of Oral Medicine and Radiology, KLE Society's Institute of Dental Sciences, Bengaluru, Karnataka, India \\ ${ }^{4}$ Reader, Department of Oral Medicine and Radiology, KLE Society's Institute of Dental Sciences, Bengaluru, Karnataka, India
}

Correspondence: S umona Pal, P rofessor, Departmentof O ral Medicine and Radiology, KLE Society's Institute of Dental Sciences No. 20 Y eshwantpur Suburb, Opposite CMTI, Tumkur Road, Bengaluru-560022, Karnataka, India, P hone: 91-9164138993 Fax: 080-23474305, e-mail: sumona_capri@yahoo.co.in

\section{ABSTRACT}

Objective: Though the soft palate presents with varied morphology, very few studies have described its diversity. The aim of our study was to investigate various shapes of soft palate in normal individuals.

Methods: The study comprised of 80 individuals requiring orthodontic treatment but without any speech abnormality, whose age ranged from 9 to 31 years. Velar shape was examined on digital lateral cephalograms and was allocated to one of the six patterns as described by You $M$ et al. The difference in proportion of each type and also difference between genders were studied.

Results: The normal soft palate can be classified into six types based on its shape. Type 2-rat-tail shape is most common in both the genders. There is no significant difference in proportion of various shapes of soft palate between genders.

Conclusion: Soft palate may have variable morphology. Knowledge of varied spectrum of velar morphology may help in successful functional and structural repair in cleft palate cases, and shed some light towards the causes of obstructive sleep apnea and related disorders.

Keywords: Soft palate, Lateral cephalograms.

\section{INTRODUCTION}

Often comprehension of normal anatomy and anomaly helps in diagnosis and successful treatment of many intricate cases. A part from evaluation of clefts, diversity in radiographic appearance of soft palate has remained unrecognized. A number of studies ${ }^{1-4}$ have been done in past towards the dimensional analysis of the soft palate and its surrounding structures, but there are not many studies regarding normal variants of soft palate morphology and configuration. Recently, Y ou M et al ${ }^{5}$ documented the variation in normal velar morphology and classified them.

The aim of our study was to investigate various shapes of soft palate on lateral cephal ograms in normal individuals.

\section{MATERIALS AND METHODS}

The sample consisted of 80 patients requiring orthodontic treatment. A total of 40 females and 40 males within the age range of 9 to 31 years formed this study group. Informed consent was taken from all the subjects. Digital lateral cephalograms were obtained using Planmeca, ProM ax (Helsinki, Finland). The subjects were stabilized in the natural head position with the mandible in maximal intercuspation and tip of tongue behind the upper central incisors, thus allowing the soft palate to simply rest on the tongue. Radiographic exposure was obtained by the machine using horizontal scanning of the patient. All radiographs were viewed using the software Dimaxis Pro 4.0.1. We employed the classification of soft palate given by $\mathrm{Y}$ ou $\mathrm{M}$ et al. ${ }^{5} \mathrm{O}$ the basis of radiographic appearances, they categorized soft pal ate into six types:

Type 1: L eaf shaped/L anceolate shaped - the middle portion of the soft palate elevated to both the naso- and the oro-side.

Type 2: R at-tail shaped - the soft pal ate which showed inflated anterior portion and free margin with an obvious coarctation.

Type3: Butt-like - soft palate which showed a shorter and fatter velum appearance with no distinct difference of width of the anterior portion to the free margin.

Type4: Straight line shaped.

Type 5: S-shaped/distorted soft palate.

Type 6: Crooked appearance- the soft palate in which the posterior portion of the soft palate crooks anteriorsuperiorly.

All the radiographs were examined and categorized by two oral radiologists separately. The weighted kappa coefficient was obtained for the two studies. A cross-tab was composed by dividing subjects into male and female groups in relation to the six categories, and Chi-square test was done to evaluate the significant relation among the variables in the tables. Significance level was based on $p$-value $<0.05$. 


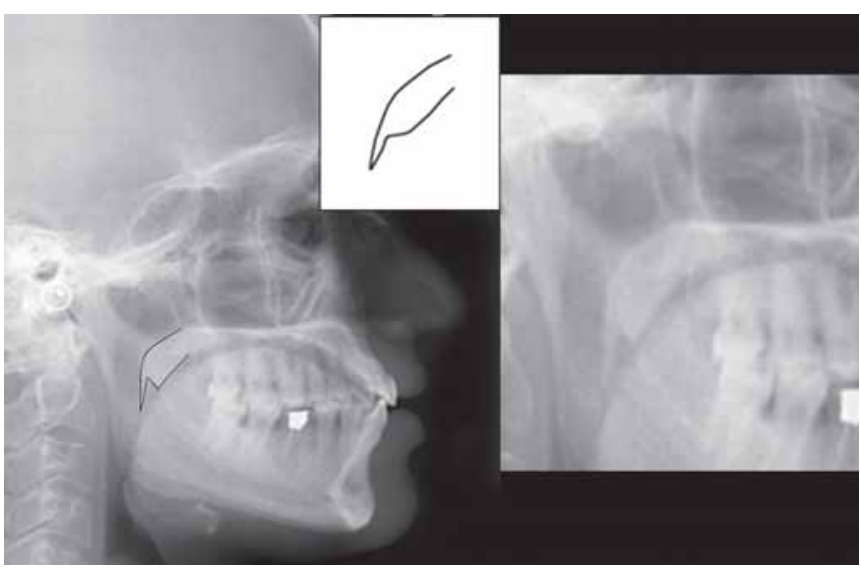

Fig. 1: Type 1-Leaf shaped

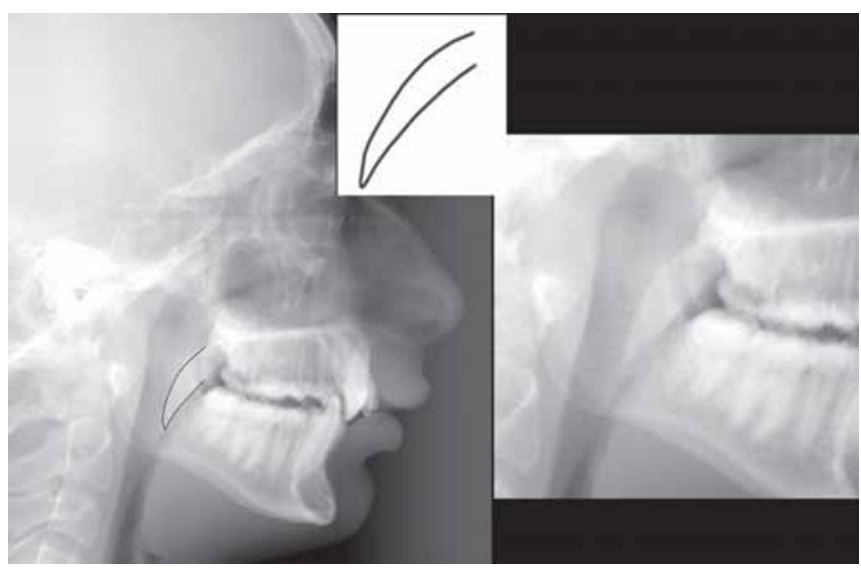

Fig. 2: Type 2-Rat-tail shaped

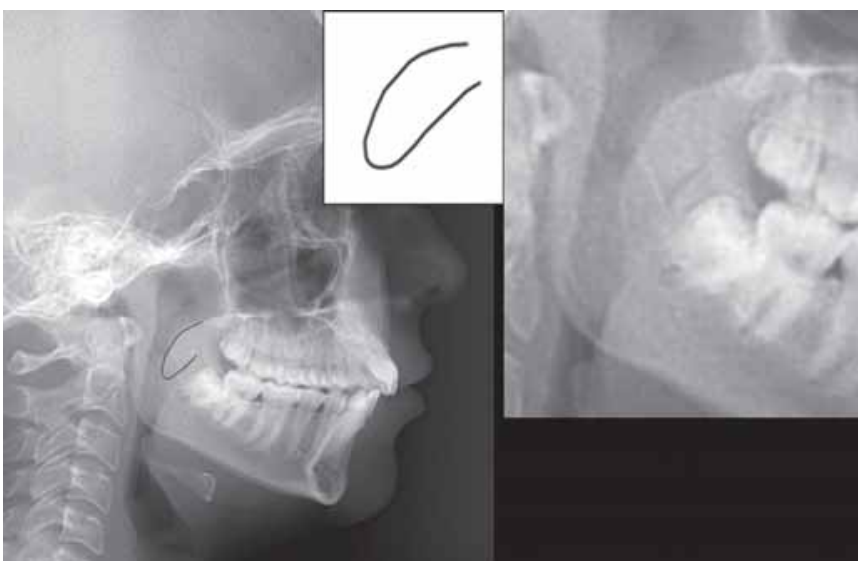

Fig. 3: Type 3-Butt-like

\section{RESULTS}

The weighted kappa value was 0.91 , i.e. in the range of nearly perfect agreement, thus reliability was consi dered to be adequate for the purpose of this study.

The distribution and proportion of various types of soft palate of our study population are shown in Tables 1 and 2 .

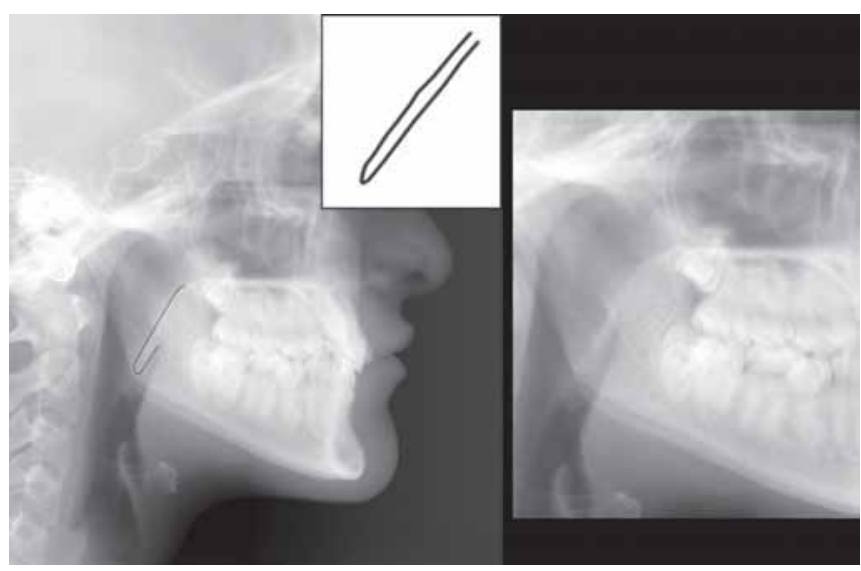

Fig. 4: Type 4-Straight line shaped

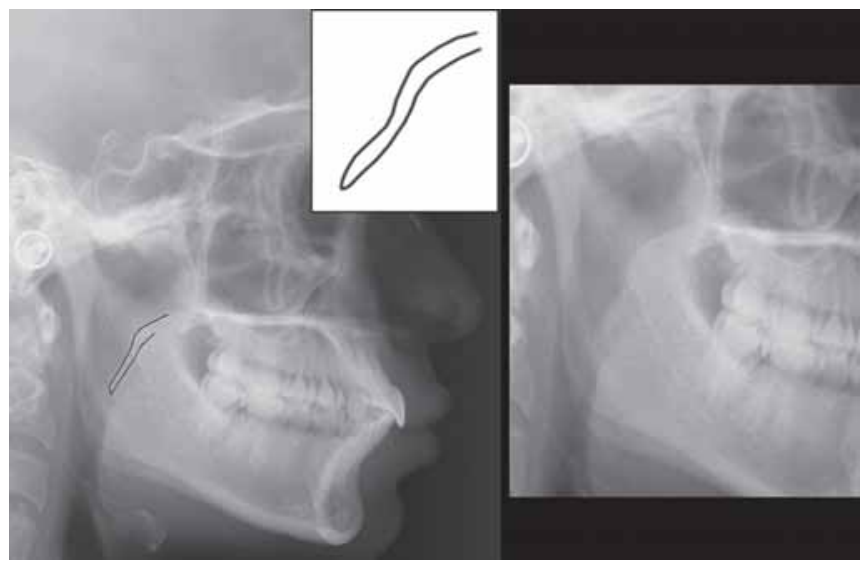

Fig. 5: Type 5-Distorted/S-shaped

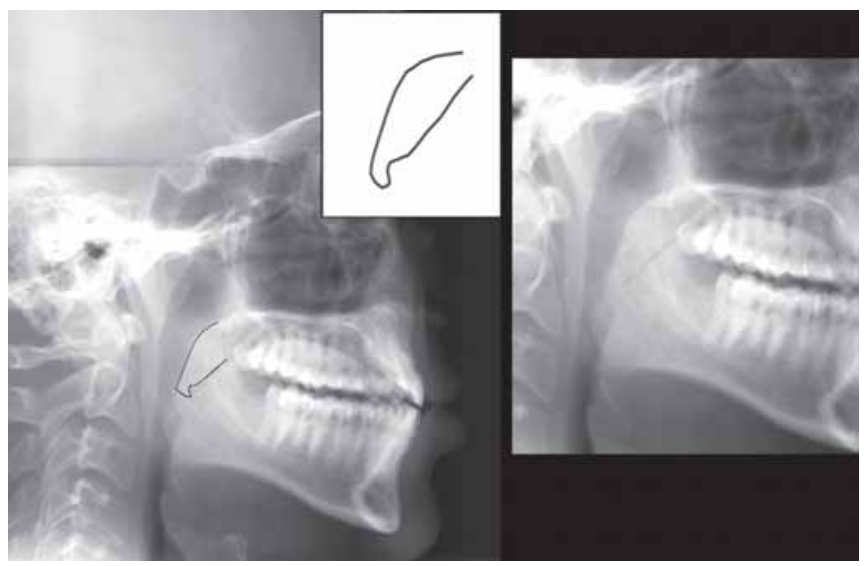

Fig. 6: Type 6-Crooked shape

A round $10 \%$ of the cases ( 2 females and 6 males) showed type 1 (Fig. 1) variety of soft palate, $55 \%$ (24 females and 20 males) had type 2 (Fig. 2) soft palates, $8.75 \%$ (1 female and 6 males) showed type 3 (Fig. 3) variety, 18.75\% (11 females and 4 males) showed type 4 (Fig. 4) patterns. Only $2.5 \%$ cases ( 1 female and 1 male) had type 5 (Fig. 5) soft palates and 5\% (1 female and 3 males) showed type 6 (Fig. 6). Type 2 soft 
Table 1: Distribution and proportion of various types of soft palate

\begin{tabular}{lrlllll} 
Type & 1 & 2 & 3 & 4 & 5 & 6 \\
\hline $\mathrm{n}$ & 8 & 44 & 7 & 15 & 2 & 4 \\
Proportion (\%) & 10 & 55 & 8.75 & 18.75 & 2.5 & 5
\end{tabular}

Table 2: Types of soft palate and gender variation

\begin{tabular}{llcc} 
Type & Female & Male & Total \\
\hline 1 & 02 & 06 & 08 \\
2 & 24 & 20 & 44 \\
3 & 01 & 06 & 07 \\
4 & 11 & 04 & 15 \\
5 & 01 & 01 & 02 \\
6 & 01 & 03 & 04 \\
\hline
\end{tabular}

palate was more prevalent than any other types. There was no significant difference of soft palate types between male and female cases. Type 5 and 6 varieties were least common.

\section{DISCUSSION}

Although advanced imaging techniques, like magnetic resonance imaging and fluoroscopy are the most effective methods for visualizing soft palate, yet lateral cephalogram remains the most common method of analysis because of its easy availability, cost effectiveness and relatively good assessment of soft tissue. Radiologic studies of soft palate morphology may greatly facilitate to diagnose a variety of neurologic, inflammatory and neoplastic disorders of the adult soft palate. The dimensional analysis of the soft palate and its surrounding structures, especially the velar length and width has been studied; neverthel ess the variety of velar morphology which is the most logical cause of different dimensions on the soft palate has been frequently overlooked. Even after closure of the soft tissue defect in cleft patients, normal function of the soft palate is frequently not achieved, and velopharyngeal insufficiency (V PI) with hypernasal speech ensues in $30 \%$ or more of patients. Cohen et al ${ }^{6}$ suggested that one of the several explanations for this surgically successful yet functionally compromised repair may be difference in morphology of soft palate and other associated structures in these patients than normal. Hence, presurgical eval uation of soft palate morphology will aid in the success of surgery.

In our study, type II shaped soft pal ate was the most frequent type ( $55 \%$ of the cases). But a previous study by $\mathrm{Y}$ ou $\mathrm{M}$ et al ${ }^{5}$ described leaf shaped soft palate to be the classic velar morphology. This variation may be due to fact that most of our study cases had malocclusion requiring orthodontic treatment.

Obstructive sleep apnea (OSA) is characterized by the recurrent occlusion of the upper airways resulting from the inspiratory collapse of the pharyngeal walls during sleep. ${ }^{6}$ Etiological or predisposing factors for OSA are still debated.
There is an increased incidence of OSA in middle aged adults. It was found that pharyngeal morphology is not immutably established during childhood and adolescence, but changes throughout adult life. There is a tendency towards longer and thicker soft palate and narrower oropharynx during adulthood, which may explain the possible increased incidence of OSA and related disorders occurring later in life. ${ }^{2}$

Pepin et $\mathrm{al}^{7}$ found that the 'hooked' appearance of the soft palate in awake patients, which indicated a high risk for obstructive sleep apnea syndrome. Y ou M et al ${ }^{5}$ found that the hooked appearance of the soft palate corresponded to S-shaped soft palate according to their classification. In our study, the type $5 \mathrm{~S}$-shaped soft pal ate was found only in two cases $(2.5 \%)$. Pepin et $\mathrm{al}^{7}$ described hooking of soft palate as angulations of about $30^{\circ}$ between the distal part of the uvula and the longitudinal axis of the soft pal ate. They hypothesized that soft palate hooking results in a sudden and major reduction in oropharyngeal dimensions, thus increasing upper airway resistance and transpharyngeal pressure gradient resulting in pharyngeal collapse. They also suggested that a hooked appearance of soft palate on images in awake patients indicates a strong possibility of obstructive sleep apnea. The success rate of uvulopalatopharyngoplasty in patients with obstructive sleep apnea has been variable. ${ }^{8}$ Pepin et $\mathrm{al}^{7}$ believed that the differentiation of patients with particular anatomic features in upper airway may be important for any future developments in successful surgical therapy for obstructive sleep apnea syndrome.

Studies reveal that there is an increase in length, thickness and sagittal area of soft palate with age ${ }^{1,3}$ in both the genders but increase in soft palate area was significantly more in males. ${ }^{7}$ In the present study, there was no significant difference between types of soft palate between males and females.

$\checkmark$ elopharyngeal closure is obtained by normal apposition of the soft palate with the posterior and lateral pharyngeal walls, thus separating the oral cavity from the nasal cavity during deglutition and speech. When the velum and lateral and posterior pharyngeal walls fail to separate the two cavities, velopharyngeal incompetence (V PI) occurs. $\mathrm{N}$ akamura et al ${ }^{9}$ reported that patients with persistent VPI had a shorter velar length and greater pharyngeal depth resulting in less than $100 \%$ length/ depth ratio, which averaged more than $100 \%$ in velopharyngeal competent subjects. $\mathrm{Y}$ ou $\mathrm{M}$ et al ${ }^{5}$ considered that a harmony between velar length and pharyngeal depth may contribute to normal velopharyngeal functions. W ada et al ${ }^{10}$ found an adequate ratio (velar length to pharyngeal depth) of about 1.3 to 1.4 in normal subjects to maintain velopharyngeal closure during speech. However, in unilateral cleft lip and cleft palate patients, it was high at an early age but gradually decreased to about 1.1 which was significantly less than the noncl eft control group at age of 17 years. The lesser value implied that the soft palate must move a greater distance to be in apposition with the posterior pharyngeal wall for attaining velopharyngeal closure. This may be the cause of reappearance of nasality at a later age in cleft palate repair patients who had a good speech earlier. 
A need ratio of $87 \%$ (computed by dividing the pharyngeal depth by the velar length at rest) is found in subjects with normal articulation and no history of cleft or palatal insufficiencies. ${ }^{11,12}$

Our study has some limitations. The stability of particular type of shape with age could not be assessed, since it is crosssectional study. Further investigations should be carried out on the changes in velar morphology with age in longitudinal study.

\section{CONCLUSION}

It can be stated that all the six types of soft palate are normal variants identifiable on lateral cephalometric radiography. $\mathrm{K}$ nowledge of varied spectrum of velar morphology may help in successful functional and structural repair in cleft palate cases, and may also be used in our drive for research into the cause of OSA and related disorders.

\section{REFERENCES}

1. Kollias I, Krogstad 0 . A dult craniocervical and pharyngeal changes: A longitudinal cephalometric study between 22 and 42 years of age: $M$ orphology of uvulo-glossopharyngeal changes (Part II). Eur J Orthod 1999;21:345-55.

2. Johnston $C D$, Richardson A. Cephalometric changes in adult pharyngeal morphology. Eur J Orthod 1999;21:357-62.

3. Taylor M, H ans M G, Strohl K P, N elson S, Broadbent B H. Soft tissue growth of the oropharynx. Angle Orthodontist 1996;66:393-400.

4. Satoh K, W ada T, Tachimura T, Shiba R. The effect of growth of nasopharyngeal structures in velopharyngeal closure in patients with repaired cleft palate and controls without clefts: A cephal ometric study. B ritish J ournal of Oral and M axillofacial Surgery 2002;40:105-09.

5. Y ou M, Li X, W ang H, Zhang J, Wu H, Liu Y, et al. M orphological variety of the soft palate in normal individuals: A digital cephalometric study. D entomaxillofac Radiol 2008;37:344-49.

6. Cohen SR, Chen L, Trotman CA, Burdi A R. Soft-palate myogenesis: A developmental field paradigm. Cleft PalateCraniofac J 1993;30:441-46.

7. Pepin J L, V eale D, Ferretti GR, M ayer P, L evy PA . Obstructive sleep apnea syndrome: Hooked appearance of the soft palate in awake patients: Cephalometric and CT findings. Radiology 1999;210:163-70.

8. Rodenstein DO. A ssessment of uvul opal atopharyngoplasty for the treatment of sleep apnea syndrome. Sleep 1992;15 (suppl 6):S56-62.

9. Nakamura N, Ogata $Y$, K unimitsu K, Suzuki A, Sasaguri M, Ohishi M. Velopharyngeal morphology of patients with persistent velopharyngeal incompetence following repushback surgery for cleft palate. Cleft Palate Craniofac J 2003;40(6): 612-17.

10. Wada T, Satoh K, Tachimura T, Tatsuta U. Comparison of nasopharyngeal growth between patients with clefts and noncleft controls. Cleft Palate Craniofac J 1997;34(5):405-09.

11. Simpson RK, A ustin A A. A cephal ometric investigation of velar stretch. Cleft Palate J 1972;9:341-51.

12. Hoopes JE, Dellon AL, Frabrikant JI. Cineradiographic definition of the functional anatomy and pathophysiology of the velopharynx. Cleft Palate J 1970;7:443-54. 